\title{
Point-of-care G6PD diagnostics for Plasmodium vivax malaria is a clinical and public health urgency
}

J. Kevin Baird ${ }^{1,2}$

\begin{abstract}
Malaria caused by Plasmodium vivax threatens over 2 billion people globally and sickens tens of millions annually. Recent clinical evidence discredits the long-held notion of this infection as intrinsically benign revealing an often threatening course associated with mortality. Most acute attacks by this species derive from latent forms in the human liver called hypnozoites. Radical cure for P. vivax malaria includes therapy aimed both at the acute attack (blood schizontocidal) and against future attacks (hypnozoitocidal). The only hypnozoitocide available is primaquine, a drug causing life-threatening acute hemolytic anemia in patients with the inherited blood disorder glucose-6-phosphate dehydrogenase (G6PD) deficiency. This disorder affects 400 million people worldwide, at an average prevalence of $8 \%$ in malaria-endemic nations. In the absence of certain knowledge regarding the G6PD status of patients infected by $P$. vivax, providers must choose between the risk of harm caused by primaquine and that caused by the parasite by withholding therapy. Resolving this dilemma requires the availability of point-of-care G6PD diagnostics practical for use in the impoverished rural tropics where the vast majority of malaria patients seek care.
\end{abstract}

Keywords: Plasmodium vivax, Hypnozoite, Relapse, Primaquine therapy, G6PD deficiency, Diagnostics, Acute hemolytic anemia

\section{Background}

Imagine the clinical importance of a diagnosis that permits safe delivery of a life-saving therapy that is also capable of taking the life of one in ten patients. That diagnosis protects patients at risk of harm by exclusion from treatment and permits the safe treatment of those not excluded. Healthcare providers in the developed world would appropriately view such diagnostic capacity as compulsory, and the clinical consequences of its absence - illness or death caused by the disease or its treatment - as overt malpractice. Over the past six decades, since licensure of primaquine for anti-relapse therapy of malaria caused by Plasmodium vivax, safe access to primaquine has been denied by the absence of capacity to diagnose

Correspondence: kbaird@eocru.org; kevin.baird@ndm.ox.ac.uk ${ }^{1}$ Eijkman-Oxford Clinical Research Unit, Jalan Diponegoro No. 69, Jakarta, Indonesia

${ }^{2}$ Centre for Tropical Medicine and Global Health, Nuffield Department of Medicine, University of Oxford, Oxford, UK glucose-6-phosphate dehydrogenase (G6PD) deficiency in the impoverished rural tropics.

Therapy of acute $P$. vivax malaria requires two distinct classes of antimalarial drugs: blood schizontocidal and hypnozoitocidal, arresting the acute attack and preventing future recurrent attacks stemming from dormant liver stages called hypnozoites, respectively. The 8 -aminoquinoline primaquine is the only therapeutic option for hypnozoitocidal treatment and it invariably provokes acute hemolytic anemia in patients having inherited G6PD deficiency. The early discoverers of G6PD deficiency as the basis of "primaquine sensitivity" in some patients (in 1956) did so with small numbers of otherwise healthy African-American men and they characterized hemolysis as mild and self-limiting [1]. Understanding of the enormous diversity of G6PD deficiency came later, including the common occurrence of variants of relatively extreme sensitivity to primaquine. One reported case of primaquine-induced acute hemolytic anemia in a 13-year-old Indonesian boy having Vanua 
Lava variant represents extreme primaquine sensitivity: After receiving the fifth of $0.5 \mathrm{mg} / \mathrm{kg}$ daily primaquine doses, he reported to clinic cyanotic, dyspnic, and icteric with pronounced hemoglobinuria; his pre-treatment hemoglobin of $12.4 \mathrm{~g} / \mathrm{dL}$ had fallen to $7.2 \mathrm{~g} / \mathrm{dL}$ at presentation, and upon admission to an intensive care unit a few hours later, it registered at $5.6 \mathrm{~g} / \mathrm{dL}$ [2]. Emergency blood transfusions in this instance likely spared the boy's life. Vanua Lava-like variants of G6PD deficiency (in terms of severity of enzymatic disability) dominate across the Mediterranean, and Southwestern, Southern, and Southeastern Asia, where about $80 \%$ of the global burden of $P$. vivax occurs [3]. Primaquine therapy against relapse in the absence of close clinical monitoring or knowledge of G6PD status should be considered inherently dangerous to any patient. Today the vast majority of patients infected by $P$. vivax have almost no access to clinical monitoring or G6PD screening.

The basis of this problem is neglect, by the medical, scientific and public health communities, the history of which has been described elsewhere [1], as has that of the neglect of $P$. vivax malaria in general [4]. The erroneous assignment of an intrinsically benign and nonlife-threatening character of $P$. vivax lies at the core of these multi-factorial and complex problems [5] causing the lack of access to therapy against relapse to have been viewed as inconsequential in endemic areas. In 1981, the malaria treatment guidelines from the World Health Organization (WHO) expressed, for example, "It is doubtful if radical treatment of vivax malaria is necessary if the patient lives in an endemic area where ... reinfection is likely" [6].

This withholding of primaquine seemed a reasoned weighing of therapeutic risk and benefit. Standard doses of primaquine against relapse causes acute hemolytic anemia in G6PD-deficient patients, sometimes leading to death [7]. Avoidance of the lethal risk of primaquine for what was perceived as a non-threatening infection dominated strategic thinking by clinical and public health communities. Likewise, the scientific community failed to view safe access to primaquine therapy as a problem in need of solving, either by replacing primaquine or developing G6PD diagnostics suited to where most malaria patients live. This status quo of the acceptability of poor access to anti-relapse therapy persisted over sixty years. Very recently, however, these communities have awoken to the need to solve the therapeutic dilemma of primaquine, G6PD deficiency and P. vivax malaria. This awareness stems from three key realizations: 1) $P$. vivax is a dangerous infection; 2 ) its hypnozoites represent a dominant source of acute attacks in endemic areas; and 3) G6PD deficiency renders attacking that reservoir with primaquine an exceedingly dangerous therapeutic endeavor.

\section{The World Health Organization responds}

In 2015, the WHO Global Malaria Program acted decisively to begin removing the barriers to primaquine access for patients infected by $P$. vivax. Both the Global technical strategy for malaria 2016-2030 [8] and the Control and elimination of Plasmodium vivax malaria: A technical brief [9] express explicitly the lethal threat of $P$. vivax malaria and the necessity of radical cure with each diagnosis of that infection. Further, recognizing the importance of G6PD diagnostics, by removing the primary barrier of access to that treatment, the WHO convened an Evidence Review Group to examine available technology and devices [10].

The only proven hypnozoitocidal (anti-relapse) drugs are 8-aminoquinolines, all of which provoke hemolytic anemia in G6PD-deficient patients [7]. Thus, identifying appropriate G6PD diagnostic devices and mobilizing them to the periphery of healthcare delivery represents the only possible short-term response to the therapeutic dilemma of $P$. vivax malaria. The clinical and public health threat imposed by the hypnozoite reservoir of that species, now residing in endemic communities almost entirely unmolested by primaquine, underpins the urgency of doing so.

\section{Hypnozoite reservoir}

Relapse behaviors by $P$. vivax vary with geographic distribution in terms of the frequency, timing and multiplicity of secondary attacks [11]. Relapse behaviors of $P$. vivax from the Southwest Pacific represent the most aggressive: almost all infected quickly suffer a first relapse (within 3 weeks of patency of the primary attack) and go on to endure five or more attacks at approximately 2-month intervals, for as late as 4 years following primary infection [12]. These relapse behaviors dominate the strains of Southeast Asia as well, and at least some strains in Africa and the Americas behave similarly. Cohorts of patients diagnosed with $P$. vivax and not treated with primaquine experience an incidence density of first relapse at four to five infections/person-year [13] - approximately the same as $P$. falciparum incidence in much of holoendemic Sub-Saharan Africa. Several studies indicate that hypnozoite-borne attacks dominate over mosquito-borne primary attacks in endemic communities $[14,15]$. This dominance largely explains the relatively poor impact of conventional methods of malaria control on endemic $P$. vivax [16]. The broad lack of access to primaquine therapy drives the continuous streaming of repeated clinical attacks and onward transmission from the hypnozoite reservoir.

\section{Point-of-care G6PD diagnostics}

The availability of G6PD diagnostic capacities practical for use in the impoverished rural tropics would protect 
the minority of residents vulnerable to primaquine therapy. However, the dominating health dividend delivered by such capacity would be realizing the enormous clinical and public health benefits of primaquine therapy for the G6PD-normal majority. Moreover, the great promise of tafenoquine, an efficacious single-dose hypnozoitocidal therapy nearing licensure [17], will also require G6PD screening against the hemolytic toxicity of that 8-aminoquinoline compound.

Experts have described the target product profile (TPP) of point-of-care G6PD diagnostics for screening prior to primaquine therapy $[10,18]$. Such a kit must be relatively inexpensive, simple to use and interpret, require no laboratory equipment or skills, be useful at ambient tropical temperatures $\left(>30^{\circ} \mathrm{C}\right)$, and require no cold chain in distribution or storage. Further, the kit must exhibit perfection in negative predictive value (NPV), effectively an estimate of its ability to allow safe administration of primaquine therapy (absence of false normal testing). The current standard for screening G6PD - the fluorescent spot test intended for laboratory use - fails on almost all of those essential criteria.

Only two point-of-care G6PD diagnostic kits are commercially available in 2015: BinaxNOW G6PD ${ }^{\mathrm{m} w}$ (Alere, Orlando, FL, USA) and CareStart ${ }^{\text {tw }}$ G6PD (Access Bio, Somerset, NJ, USA). BinaxNOW G6PD ${ }^{\mathrm{Tm}}$ performs satisfactorily [19-21], but it fails the TPP in terms of cost (approximately \$15/test) and ambient tropical temperature usage (use above $25^{\circ} \mathrm{C}$ strictly prohibited by the manufacturer). CareStart ${ }^{\mathrm{mu}}$ G6PD has thus far met essential cost (\$1.50/test) and performance characteristics [22-25]. BinaxNOW G6PD ${ }^{\mathrm{mm}}$ has been registered and licensed with the US Food Drug Administration, but CareStart ${ }^{\mathrm{tm}}$ G6PD has not, and neither product is registered as a prequalified diagnostic device by the WHO. This imposes a significant barrier to kit access by national malaria control programs.

Those two commercial products no doubt represent the leading edge of point-of-care G6PD diagnostics yet to come. Both emerged from private entrepreneurial initiative and capital rather than a supported research agenda bearing development costs and risk. Public investments in research on G6PD diagnostics would no doubt yield improved technologies and devices suited to this specific application guided by a critical TPP. For the present, no other kits exist, however, and awaiting improvements would deny very many millions suffering $P$. vivax malaria safe access to primaquine therapy. That should not be considered an ethically grounded option.

\section{Conclusions}

The availability of devices permitting safe and effective therapy against a life-threatening infection by an otherwise dangerous drug should be heralded as a great stride forward in mitigating the harm done by $P$. vivax or its therapy with primaquine. The ability of national malaria control programs to adopt and integrate G6PD screening capacity into their healthcare delivery systems (to the very periphery) hinges upon the endorsement, encouragement and direct support of international agencies and civil societies. Some authorities, however, consider the point-ofcare G6PD diagnostics inadequately vetted or validated and passively await additional data. This posture seems to disregard the current standard of care for G6PD diagnostics in the impoverished rural tropics - the malpractice of no capacity whatsoever.

Understanding this problem requires acknowledging a deeply harmful status quo for chemotherapy of $P$. vivax malaria that must be actively and thoroughly discarded. Aggressively rolling out practical point-of-care G6PD diagnostics to the peripheries of healthcare delivery directly enables acting upon the WHO "call-to-arms" against the hypnozoite reservoir. In light of the clinical and public health stakes, the available data on the CareStart ${ }^{\mathrm{m}}$ G6PD kit and its satisfactory fit to the expert TPP should embolden the decisions and actions needed to mobilize it to where needed as matter of urgency. Broad success at doing so promises otherwise inconceivable gains against the global burdens of morbidity and mortality imposed by a pernicious $P$. vivax problem.

\section{Abbreviations}

G6PD: glucose-6-phosphate dehydrogenase; NPV: negative predictive value; TPP: target product profile; WHO: World Health Organization.

\section{Competing interests}

JKB has received G6PD test kits, equipment and technical advice in their use from Access Bio (Somerset, NJ, USA), and publishes findings without financial reward, obligation or restriction from that corporation. JKB holds no financial stake in Access Bio or any of its products, and does not hold any patents or other financial stake in products relating to G6PD diagnostics or antimalarial therapeutics. JKB served as an unpaid technical advisor for the WHO on steering and technical committees, drafting the documents cited as references 8,9 and 10. JKB's laboratory receives research support funding from GlaxoSmithKline (Cambridge, UK) for clinical trials of tafenoquine against relapse of $P$. vivax.

\section{Acknowledgements}

The Wellcome Trust provided JKB with support, with grant number B9RJIXO

Received: 19 November 2015 Accepted: 20 November 2015

Published online: 14 December 2015

\section{References}

1. Baird JK. Origins and implications of neglect of G6PD deficiency and primaquine toxicity in Plasmodium vivax malaria. Pathog Glob Health. 2015;109:93-106.

2. Satyagraha AW, Sadhewa A, Baramuli V, Elvira R, Ridenour C, Elyazar I, et al. G6PD deficiency at Sumba in eastern Indonesia is prevalent, diverse, and severe: implications for primaquine therapy against relapsing vivax malaria. PLoS Negl Trop Dis. 2015;9:e0003602.

3. Howes RE, Piel FB, Patil AP, Gething PW, Dewi M, Hogg MM, et al. G6PD deficiency prevalence and estimates of affected populations in malaria endemic countries: a geostatistical model-based map. PLoS Med. 2012;9: e1001339

4. Price RN, Tjitra E, Guerra CA, Yeung S, White NJ, Anstey NM. Vivax malaria: neglected and not benign. Am J Trop Med Hyg. 2007;77(6 Suppl):79-87. 
5. Baird JK. Evidence and implications of mortality associated with acute Plasmodium vivax malaria. Clin Microbiol Rev. 2013;26:36-57.

6. Bruce-Chwatt LJ, Black RH, Canfield CJ, Clyde DF, Peters W, Wernsdorfer WH. Chemotherapy of malaria. 2nd ed. Geneva: World Health Organization; 1981.

7. Recht J, Ashley E, White N. Safety of 8-aminoquinoline antimalarial medicines. Geneva: World Health Organization; 2014.

8. World Health Organization. Global technical strategy for malaria 2016-2030. Geneva: World Health Organization; 2015.

9. World Health Organization. Control and elimination of Plasmodium vivax malaria. A technical brief. Geneva: World Health Organization; 2015.

10. World Health Organization. Point-of-care G6PD testing to support safe use of primaquine for the treatment of vivax malaria. WHO Evidence Review Group meeting report, 8-9 October 2014, WHO/UNAIDS Building, Geneva, Switzerland. Geneva: World Health Organization; 2015.

11. Battle KE, Karhunen MS, Bhatt S, Gething PW, Howes RE, Golding N, et al. Geographical variation in Plasmodium vivax relapse. Malaria J. 2014;13:144.

12. Hill $E$, Amatuzio DS. Southwest Pacific vivax malaria: clinical features and observations concerning duration of clinical activity. Am J Trop Med Hyg. 1949;29:203-14.

13. Sutanto I, Tjahjono B, Basri H, Taylor WR, Putri FA, Meilia RA, et al. Randomized, open-label trial of primaquine against vivax malaria relapse in Indonesia. Antimicrob Agents Chemother. 2013;57:1128-35.

14. White MT, Karl S, Battle KE, Hay SI, Mueller I, Ghani AC. Modelling the contribution of the hypnozoite reservoir to Plasmodium vivax transmission. Elife. 2014; 3. doi: 10.7554/eLife.04692

15. Roy M, Bouma MJ, Ionides EL, Dhiman RC, Pascual M. The potential elimination of Plasmodium vivax malaria by relapse treatment: insights from a transmission model and surveillance data from NW India. PLoS Negl Trop Dis. 2013;7:e1979.

16. Maude RJ, Nguon C, Ly P, Bunkea T, Ngor P, de la Torre SE C, et al. Spatial and temporal epidemiology of clinical malaria in Cambodia 2004-2013. Malaria J. 2014;13:385.

17. Llanos-Cuentas A, Lacerda MV, Rueangweerayut R, Krudsood S, Gupta SK Kochar SK, et al. Tafenoquine plus chloroquine for the treatment and relapse prevention of Plasmodium vivax malaria (DETECTIVE): a multicentre, double-blind, randomised, phase $2 \mathrm{~b}$ dose-selection study. Lancet. 2014;383:1049-58.

18. Domingo GJ, Satyagraha AW, Anvikar A, Baird JK, Bancone G, Bansil P, et al. G6PD testing in support of treatment and elimination of malaria: recommendations for evaluation of G6PD tests. Malaria J. 2013;12:391.

19. Osorio L, Carter N, Arthur P, Bancone G, Gopalan S, Gupta SK, et al. Performance of BinaxNOW G6PD deficiency point-of-care diagnostic in $P$. vivax-infected subjects. Am J Trop Med Hyg. 2015;92:22-7.

20. Tinley KE, Loughlin AM, Jepson A, Barnett ED. Evaluation of a rapid qualitative enzyme chromatographic test for glucose-6-phosphate dehydrogenase deficiency. Am J Trop Med Hyg. 2010;82:210-4.

21. LaRue N, Kahn M, Murray M, Leader BT, Bansil P, McGray S, et al. Comparison of quantitative and qualitative tests for glucose-6-phosphate dehydrogenase deficiency. Am J Trop Med Hyg. 2014;91:854-61.

22. Baird JK, Dewi M, Subekti D, Elyazar I, Satyagraha AW. Non-inferiority of glucose-6-phosphate dehydrogenase deficiency diagnosis by a point-of-care rapid test vs the laboratory fluorescent spot test demonstrated by copper inhibition in normal human red blood cells. Transl Res. 2015;165:677-88.

23. Adu-Gyasi D, Asante KP, Newton S, Dosoo D, Amoako S, Adjei G, et al. Evaluation of the diagnostic accuracy of CareStart G6PD deficiency Rapid Diagnostic Test (RDT) in a malaria endemic area in Ghana, Africa. PLoS One. 2015;10:e0125796.

24. von Fricken ME, Weppelmann TA, Eaton WT, Masse R, de Rochars MV B, Okech BA. Performance of the CareStart glucose-6-phosphate dehydrogenase (G6PD) rapid diagnostic test in Gressier, Haiti. Am J Trop Med Hyg. 2014;91:77-80.

25. Roca-Feltrer A, Khim N, Kim S, Chy S, Canier L, Kerleguer A, et al. Field trial evaluation of the performances of point-of-care tests for screening G6PD deficiency in Cambodia. PLoS One. 2014;9:e116143.

\section{Submit your next manuscript to BioMed Central and we will help you at every step:}

- We accept pre-submission inquiries

- Our selector tool helps you to find the most relevant journal

- We provide round the clock customer support

- Convenient online submission

- Thorough peer review

- Inclusion in PubMed and all major indexing services

- Maximum visibility for your research

Submit your manuscript at www.biomedcentral.com/submit 